\title{
Serological and parasitological response in chronic Chagas patients 3 years after nifurtimox treatment
}

\author{
Yves Jackson ${ }^{1 *}$, Eric Chatelain ${ }^{2}$, Anne Mauris ${ }^{3}$, Marylise Holst ${ }^{1}$, Qianqian Miao ${ }^{4}$, Francois Chappuis ${ }^{5}$ \\ and Momar $\mathrm{NdaO}^{4}$
}

\begin{abstract}
Background: With declining vectorial transmission, Chagas disease predominantly affects adults nowadays. The efficacy of nifurtimox in the chronic phase in adult patients is poorly known, particularly in regions where there is no risk of reinfection. Recommendations for treatment outcome assessment rely on serological follow-up. We evaluated the serological and parasitological response to nifurtimox in a cohort of adult patients three years post-treatment in Switzerland.

Methods: Patients treated with nifurtimox in 2008 during a cross-sectional study in Geneva, Switzerland, were contacted for follow-up in 2011. Two ELISAs and a rapid immunochromatographic test were used to test 2008 and 2011 serum samples simultaneously. In addition, conventional and real-time PCR were performed on 2011 samples.

Results: Thirty-seven (84.1\%) of 44 eligible patients, predominantly female, middle-aged, Bolivians at the indeterminate stage, were enrolled. All 2011 ELISA and immunochromatographic tests were positive. Twenty-eight (75.7\%) patients presented a lower optical density (OD) in 2011 compared to 2008. This OD difference was significant in both commercial $(P<0.001)$ and in-house $(P=0.002)$ ELISAs. Agreement between the two ELISAs was low (Kappa $=0.469)$. All patients had negative conventional PCR results but one $(2.7 \%)$ was positive with real-time PCR.

Conclusion: Our results highlight the inadequacy of serology for assessing response in adults, three years after treatment. In our cohort, $97.3 \%$ had results that could either indicate treatment failure or persistant humoral response despite treatment. The lack of accurate early post-treatment tests of cure prevents appropriate patients information and councelling. New follow-up tests are needed to assess treatments efficacy given the large adult population in need of antiparasitic therapy.
\end{abstract}

Keywords: Chagas disease, Trypanosoma cruzi, Diagnostic tests, Follow-up, Serology, PCR, Rapid diagnostic tests

\section{Background}

Chagas disease affects $8-10$ million people in Latin America and up to 80,000 and 300,000 in Europe and in the United States respectively, two areas free from vectorial transmission $[1,2]$. With declining rates of vectorial infection, the burden of this chronic disease is now predominantly carried by adults [3]. Recent guidelines

\footnotetext{
* Correspondence: yves.jackson@hcuge.ch

'Division of primary care medicine, Department of community medicine, primary care and emergency medicine, Geneva University Hospitals and University of Geneva, Geneva, Switzerland

Full list of author information is available at the end of the article
}

and practices in non-endemic countries extended treatment indications to adults at the indeterminate phase or with mild cardiopathy, to prevent or mitigate potentially lethal cardiac damage $[4,5]$. Benznidazole and nifurtimox are the only antiparasitic drugs registered for this indication. The significant side-effects and drop-out rates in adults raises safety and efficiency concerns about the large scale implementation of adult treatment programmes [6,7].

The main criterion for treatment success is return to sereonegativity against T.cruzi [8]. To date, most post treatment serological studies in adults have been conducted in

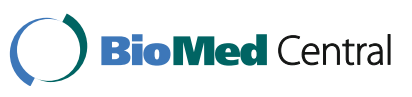


patients treated with benznidazole [9]. This drug is frequently recommended by experts as the first line treatment due to its greater tolerance, even though no randomized trial has ever evaluated the comparative safety and efficacy of nifurtimox and benznidazole in adults $[7,10]$. There has been no follow-up study of nifurtimox treated patients in non endemic regions, where the persistance of a seropositive result cannot be attributed to vectorial reinfection.

Recently, rapid diagnostic tests have proved both sensitive and highly specific in screening adults for Chagas disease $[11,12]$. They facilitate the screening of hard-to-reach populations such as rural communities or migrants in conditions where access to laboratory is difficult. Conventional serologies should be used to confirm the diagnosis. No study has evaluated their use as a follow-up tool posttreatment. Conventional polymerase chain reaction (PCR) has been found to lack sensitivity in adults at the chronic phase of the disease when used as a first-line diagnostic test [13]. This results from the low and intermittent circulating parasitic load in adults during the chronic phase. No information is available about PCR in adults following nifurtimox treatment.

This study aimed to observe the changes in conventional and rapid serological diagnostic tests in a cohort of adults living in a non-endemic country after nifurtimox treatment. The presence of circulating $T$. cruzi detected by conventional and real-time PCR in this cohort was also examined. We found that all follow-up serological tests three years after treatment remained positive with one patient having a positive real-time PCR. It means that $97.3 \%$ of patients could not be classified as either suffering treatment failure or having persistant humoral response despite treatment.

\section{Methods}

\section{Ethical statement}

The protocol of this study was approved by the Ethical Board for Medical Research of the Geneva University Hospitals (protocol 11-162). Written informed consent was obtained from all participants.

\section{Study design and setting}

This cohort study took place at the Geneva University Hospitals, which are the reference center for the screening and clinical management of Chagas disease in Geneva, Switzerland. Geneva hosts a substantial population of Latin American migrants originating from endemic areas for Chagas disease. Migrants' communities and support groups were informed about the study.

\section{Study population}

In 2008, we diagnosed Trypanosoma cruzi infection in 130 of 1012 (12.8\%) adult Latin American migrants participating in a community-based cross-sectional study in Geneva,
Switzerland [14]. The diagnosis was based on positive results in two different serological tests (BioMerieux Elisa cruzi ${ }^{\circledR}$ and the Biokit Bioelisa Chagas ${ }^{\circledR}$ ). All participants with positive results were also tested with a rapid diagnostic test $\left(\right.$ Stat-Pak $\left.{ }^{\circledR}\right)$. At that time, no PCR was performed to search for circulating T. cruzi. The 130 cases were at the chronic phase of the infection with 14 (11.3\%) and $1(0.8 \%)$ showing evidence of $T$. cruzi cardiac and digestive damage, respectively. Eighty-one participants started treatment with nifurtimox $(10 \mathrm{mg} / \mathrm{kg} /$ day $)$, however only 41 of these (56.2\%) completed the 60 -day treatment. Participants provided informed consent to have serum samples stored for future Chagas disease diagnostic studies. Sera were frozen $\left(-20^{\circ} \mathrm{C}\right)$ upon collection and stored in a bio-bank at the Geneva University Hospitals.

\section{Study participants}

From October $3^{\text {rd }}$ to November $30^{\text {th }} 2011$, patients diagnosed with Chagas disease and who started nifurtimox treatment during the 2008 study and still living in Geneva ( $\mathrm{n}=44$ ) were invited to participate. Patients were contacted by phone by the study nurse. In the event of five unanswered phone calls, an invitation letter was sent to the last recorded address. Study information, consent form and convocation letters were written in Spanish to enhance participation.

\section{Samples collection}

A qualified nurse collected two blood samples from each participant's cubital vein: one tube containing EDTA for PCR and one tube without anticoagulant for serological tests, the latter being centrifuged and aliquoted in three subsamples. All samples were stored at $-20^{\circ} \mathrm{C}$ within 1 hour upon collection. One frozen serum aliquot per participant was sent to the National Reference Center for Parasitology, Research Institute of the McGill University Health Centre, Montreal General Hospital, Canada for in house ELISA and PCR testing.

\section{Patient characteristics}

Sociodemographic characteristics were collected by the study nurse at the time of the blood sample collection. Information was cross-checked with those from the 2008 study. Clinical information on the stage of the disease, 2008 serum rapid immunochromatographic assay results and details about anti-trypanosomal therapy was extracted from the 2008 study files.

\section{Serological follow-up}

In Geneva, the Stat-Pak ${ }^{\circledR}$ was performed on one of the serum aliquots according to the manufacturer's recommendations. Results were recorded as positive or negative. After retrieving one 2008 serum aliquot for each participant, the 2008 and 2011 serum samples were tested with 
the Biokit Bioelisa Chagas ${ }^{\circledR}$ ELISA. All tests were done simultaneously using the same batch of tests and on the same machine to reduce random variability in measurements. In Montreal, all 2008 and 2011 samples were simultaneously tested with an in-house ELISA using fixed forms of Trypanosoma cruzi (epimastigotes, amastigotes, and trypomastigotes) as described elsewhere [15]. The cut-off value of the optical density (OD) for negativity of the Biokit Bioelisa Chagas ${ }^{\circledR}$ was 0.320 , as instructed by the manufacturer and 0.4 for the in-house ELISA. Results were reported as a continuous variable for the measurement of $\mathrm{OD}$ values and as a categorical variable for the final result (positive versus negative). No cut-off for clinical significance was set for the interpretation of the quantitative OD value difference in absence of valid evidences and following the manufacturer's recommendation.

\section{PCR assays}

DNA extraction was performed from whole blood samples using QIAamp DNA Mini Kit (Qiagen, San Diego, CA) following the manufacturer's instructions. Conventional PCR was performed as described by Ndao et al. [16]. LightCycler PCR detection and analysis systems (LightCycler 1.5, Roche Applied Science, Manheim, Germany) were used for amplification and quantification. The reaction was performed in glass capillaries. T. cruzi specific primers TCZ-3 5'-TGC ACT CGG CTG ATC GTT T-3'and TCZ-4 $5^{\prime-}-A T T$ CCT CCA AGC AGC GGA TA-3'(Ndao et al.) [16] and probe TcruziTM5'-6FAM-TGC TGC ATC ACA CGT TGT GGT CCA-BBQ-3' (TIB MOLBIOL, Adelphia, NJ), were designed to amplify the parasitic genomic DNA. For amplification detection, the LightCycler $^{\mathrm{TM}}$ Fastart TaqMan ${ }^{\circledR}$ Master Kit (Roche Applied Science) was used as recommended by the manufacturer. Briefly, a total of $20 \mu \mathrm{l}$ of reaction consists of $2 \mu \mathrm{l}$ each of primers TCZ-3 and TCZ-4 (1 $\mu \mathrm{M}$ final concentration), $2 \mu \mathrm{l}(0.4 \mu \mathrm{M})$ of Tcruzi TM, $4 \mu \mathrm{l}$ of $5 \mathrm{x}$ reaction buffer mix and $5 \mu \mathrm{l}$ distilled $\mathrm{H}_{2} \mathrm{O}$. Each sample was analysed in triplicate, and $5 \mu \mathrm{l}$ of DNA sample was added in each capillary. The qPCR reaction was performed with the cycle condition of 1 cycle at $95^{\circ} \mathrm{C}$ for $10 \mathrm{~min}, 45$ cycles of $95^{\circ} \mathrm{C}$ for $10 \mathrm{~s}, 61^{\circ} \mathrm{C}$ for $8 \mathrm{~s}$, and $72^{\circ} \mathrm{C}$ for $20 \mathrm{~s}$ and 1 final cycle of $40^{\circ} \mathrm{C}$ for $30 \mathrm{~s}$. The cycle number, determined by the melting curve, was presented as the number of PCR cycles needed for the fluoresce signal of the amplified products to exceed the detected threshold value.

\section{Statistical analysis}

Continuous non-normally distributed variables are presented with median and interquartile range (IQR). We used the parametric paired $t$-test and the non-parametric Wilcoxon rank sum test to compare 2008 and 2011 OD values. Fischer's exact and McNemar's tests were used for comparison of independent and paired proportions. The significance level was set at $0.05 \%$. Analyses were conducted using SPSS software, version 20.

\section{Results}

\section{Patients}

Of the 44 eligible patients, 37 (84.1\%) were enrolled in the study, while $3(6.8 \%)$ refused to participate and 4 (9.1\%) could not be reached. Table 1 shows the sociodemographic and clinical characteristics of the 37 participants. The 7 non participants had similar characteristics (data not shown).

\section{ELISA and rapid immunochromatographic tests}

All 37 post-treatment results were positive with the two ELISAs and the rapid immunochromatographic test (Table 2). Twenty-eight (75.7\%) participants had decreased ELISA OD values in 2011 compared to 2008 with significantly lower OD values in both the Biokit Bioelisa Chagas $(\mathrm{P}<0.001)$ and the in-house ELISA $(\mathrm{P}=0.002)$. Agreement between the two tests was low (Kappa $=0.469$ ). There was no significant association between the proportions of patients with reduced OD value in 2011 and gender $(P=0.66)$, age $(P=0.45)$, stage of disease $(P=1)$ and treatment duration $(\mathrm{P}=0.69)$.

\section{PCR testing}

Conventional PCR testing was negative in all 37 patients, whereas real-time PCR was positive in 1 (2.7\%) patient.

\section{Discussion}

This study shows that none of two ELISAs and a rapid immunochromatographic test $\left(\right.$ Stat-Pak ${ }^{\circledR}$ ) reverted to negativity three years after nifurtimox treatment in a cohort of adults at the chronic stage of Chagas disease living in an area without vectorial transmission. One patient was found positive by Real-Time PCR. Therefore, 97.3\% participants could not be adequately informed about their current clinical status, with a single patient conclusively found to be still infected. The positive serological test results could either indicate treatment failure or simply reflect the window period of persistent humoral response despite potential treatment efficacy. Moreover, we found low agreement between the two ELISAs in regards to decreasing OD values post treatment. Whereas the main limitation of the study is the sample size of 37 participants, it is the largest serological follow-up study of adults treated with nifurtimox published to date and the first study carried out in a non-endemic country.

To date, there is no early marker of treatment efficacy in adults. Currently, the criterion of treatment success is the return to negativity of a previously positive serological test and it is recommended to undergo a 10- to 20-year long serological follow-up to assess treatment efficacy in adults [8]. These recommendations are more appropriate for the 
Table 1 Sociodemographic and clinical characteristics of the 37 participants

\begin{tabular}{lll}
\hline \multirow{2}{*}{ Gender } & Male & $\begin{array}{l}\text { N (\%) or } \\
\text { median (range) }\end{array}$ \\
\cline { 2 - 3 } & Female & $7(18.9)$ \\
\hline Age (years) & & $30(81.1)$ \\
\hline Origin & Bolivia & $44(25-59)$ \\
\cline { 2 - 3 } & Argentina & $1(2.7)$ \\
\hline Staging & Indeterminate & $31(83.8)$ \\
\cline { 2 - 3 } & Cardiopathy & $6(16.2)$ \\
\hline Duration of treatment (days) & 60 & $25(67.6)$ \\
\cline { 2 - 3 } & $30-59$ & $4(10.8)$ \\
\cline { 2 - 3 } & $<30$ & $8(21.6)$ \\
\hline
\end{tabular}

management of children, whose serology usually return to negativity within months. Our findings highlight the lack of utility of these recommendations for adults in clinical settings as all qualitative serological results remained unchanged 3 years after treatment with nifurtimox.

These results, in patients with no risk of vectorial reinfection following treatment, confirm the long lasting anti-T. cruzi humoral response after treatment, which can persist for more than 20 years [17-20]. In addition to showing this long window period, these studies in adults treated with benznidazole and nifurtimox found overall low $(<50 \%)$ seronegativation rates. There is evidence that a seronegative response following benznidazole treatment is correlated with a reduced risk of cardiac damage and possibly of mortality $[17,20,21]$. Few studies have specifically evaluated serological response following nifurtimox treatment in adults. Fabbro and colleagues followed a cohort of 27 adults over 21 years in Argentina. They found a trend towards a higher rate of seronegativation in those treated with nifurtimox (40.5\%) than with benznidazole (33.3\%) [17]. Coura et al., found no serological change in 19 Brazilian patients, one year after nifurtimox treatment [18]. In our cohort, there was no way to reliably discriminate between treatment failure and latency before seronegativation, except for the single patient with a positive PCR indicating treatment failure. Moreover, the low agreement between tests regarding OD kinetic tends to demonstrate the lack of utility of this potential criterion of cure. We observed a significant decrease in optical density levels in both ELISAs tests in $75.7 \%$ patients. The exact significance of this finding is difficult to assess, but a non-randomized study performed following treatment with benznidazole showed a positive correlation between declining antibody titers and a reduction in the risk of cardiac damage [20]. In Argentina, Fabbro et al. showed that changes in antibody titers occurred 7 years or later after nifurtimox treatment [17]. The ongoing BENEFIT cohort study will provide additional information on the serological kinetic and the protective effect of benznidazole - but not nifurtimox - in adults [22].

No follow-up study has addressed the evolution of rapid diagnostic test results after treatment. In our study population, the Stat-Pak ${ }^{\circledR}$ rapid immunochromatogaphic test has proved reliable at the diagnostic stage [11]. However, after three years, all rapid tests remained positive, which is consistent with ELISA results. Therefore, the Stat-Pak ${ }^{\circledR}$ cannot be recommended as a test of cure, at least in the three year post-treatment period.

Different PCR techniques and primers are used to detect circulating T.cruzi. Overall, they are not appropriate to diagnose the chronic stage of infection due to limited (60-90\%) sensitivity, technical challenges and lack of standardization $[10,13,23]$. However, PCR is useful in verifying infection where there are contradictory serological results, and to confirm treatment failure where there are persistent seropositive results following treatment. Realtime PCR is considered more sensitive for parasite detection than conventional methods and therefore may be a better tool to assess treatment failure [24]. In this study, we used two PCR techniques and found a discordant result in one $(2.7 \%)$ patient and concordant negative results in all others. In the absence of pre-treatment PCR measurements, we were not able to identify changes. PCR is constantly evolving and efforts are underway to standardize methods [13]. Further studies are needed to assess their exact role as a test of cure.

The limited efficacy of currently available treatments and the lack of rapidly responsive tests of cure have a significant impact on the clinical management of adults and on public health. In our experience, the inability of clinicians to propose rapid and appropriate information on the treatment outcome soon after its completion may create some resistance in patients during the pre-treatment information session. Some of our patients have been reluctant to start a

Table 2 Results of ELISA, rapid immunochromatographic and PCR (conventional and real-time) tests in 37 patients with Chagas disease, three years after treatment with nifurtimox

\begin{tabular}{|c|c|c|c|c|c|}
\hline & Biokit Bioelisa Chagas $^{\boxplus}$ & In-house ELISA & Stat-Pak & Conventional PCR & Real time $P C R$ \\
\hline & $n(\%)$ & $\overline{n(\%)}$ & $n(\%)$ & $n(\%)$ & $n(\%)$ \\
\hline Positive & $37(100)$ & $37(100)$ & $37(100)$ & $0(0)$ & $1(2.7)$ \\
\hline Negative & $0(0)$ & $0(0)$ & $0(0)$ & $37(100)$ & $36(97.3)$ \\
\hline Inconclusive & $0(0)$ & $0(0)$ & $0(0)$ & $0(0)$ & $0(0)$ \\
\hline
\end{tabular}


long and potentially poorly tolerated treatment in absence of a rapidly measurable outcome. Moreover, clinicians are unable to adequately inform treated patients on their subsequent risk of cardiopathy as long as the tests remain positive. This hampers personalized counseling on followup and forces patients to undergo serial serological tests over years. Today's patients are highly mobile and Chagas disease has become a global health problem [25]. International migrants with Chagas disease, amounting to half a million at least, may receive treatment in one country and subsequently move to another, making the currently recommended long-term serological follow-up highly impracticable [25]. Moreover, given the frequent presence of other modifiable cardiac risk factors in adults with Chagas disease, the lack of accurate post-treatment evaluation strategies hamper clinicians in differentiating between possible causes of subsequent cardiac damage [26]. The reliance on the return to negativity of serologies as a proof of treatment success means that treated patients are denied the opportunity to donate blood and organs for many years until tests may eventually become negative. This is unfortunate as Latino American migrants are more willing to donate blood than local residents in Switzerland [14]. Finally, new treatment evaluation is made difficult in absence of early marker of cure.

\section{Conclusions}

In conclusion, currently recommended strategies to assess treatment outcome in adults, at least in those receiving nifurtimox, are useless in clinical setting given the extremely long time-frame necessary to identify change, and rapid diagnostic tests seem not to offer a valid alternative. In addition to searching for reactive antigens, new diagnostic techniques such as proteomics, flow cytometry and the monitoring of $T$. cruzi specific $T$ cells response are currently under investigation and will hopefully improve the early assessment of treatment outcome [27-30]. The management of adult Chagas disease needs rapid progress. More efficient and safer treatments, together with accurate and early post-treatment tests of cure, will bring about a giant leap forward in the clinical management of millions of adult patients.

\section{Abbreviations}

PCR: Polymerase chain-reaction; ELISA: Enzyme linked immunosorbent assay; EDTA: Ethylene Diamine Triacetic Acid; OD: Optical density.

\section{Competing interests}

The authors declare that they have no competing interests.

\section{Authors' contribution}

YJ conceived the study, performed the statistical analysis and wrote the manuscript. EC conceived the study, wrote the protocol, reviewed the analysis and proofread the manuscript. AM performed the serological analysis and proofread the manuscript. $\mathrm{MH}$ gathered the samples and proofread the manuscript. MQ performed the serologies and the PCR and proofread the manuscript. FC conceived the study and proofread the manuscript. MN performed the serologies and the PCR and proofread the manuscript. All authors read and approved the final manuscript.

\section{Acknowledgements}

This study was supported by a grant from the Drugs for Neglected Diseases initiative in Geneva. DNDi is grateful to its donors, public and private, who have provided funding since its inception in 2003. A full list of DNDi's donors can be found at http://www.dndi.org/index.php/donors.html?ids=8.

For the work described in this paper, DNDi allocated non-earmarked funding (Department for International Development (DFID)/United Kingdom, Spanish Agency for International Development Cooperation (AECID)/Spain, Swiss

Agency for Development and Cooperation (SDC)/Switzerland, Médecins Sans Frontières (Doctors without Borders)/International). The donors had no role in study design, data collection and analysis, decision to publish, or preparation of the manuscript.

\section{Author details}

${ }^{1}$ Division of primary care medicine, Department of community medicine, primary care and emergency medicine, Geneva University Hospitals and University of Geneva, Geneva, Switzerland. ${ }^{2}$ Drug for Neglected Diseases Initiative, Geneva, Switzerland. '3epartment of genetics and laboratory analysis, Geneva University Hospitals and University of Geneva, Geneva, Switzerland. ${ }^{4}$ National Reference Center for Parasitology, Department of Medicine, Division of Infectious Diseases, Research Institute of the McGill University Health Centre, Montreal General Hospital, Montreal, Canada. ${ }^{5}$ Division of international and humanitarian medicine, Department of community medicine, primary care and emergency medicine, Geneva University Hospitals and University of Geneva, Geneva, Switzerland.

Received: 13 September 2012 Accepted: 8 February 2013

Published: 13 February 2013

\section{References}

1. Bern C, Montgomery SP: An estimate of the burden of Chagas disease in the United States. Clin Infect Dis 2009, 49(5):e52-e54.

2. World Health Organization: Control and prevention of Chagas disease in Europe. Report of a WHO Informal Consultation (jointly organized by WHO headquarters and the WHO Regional Office for Europe) Geneva, Switzerland, 17-18 December 2009. Geneva: WHO; 2009.

3. World Health Organization: Working to overcome the global impact of neglected tropical diseases: first WHO report on neglected tropical diseases. Geneva: WHO; 2010.

4. Coura JR, Borges-Pereira J: Chronic phase of Chagas disease: why should it be treated? A comprehensive review. Mem Inst Oswaldo Cruz 2011, 106(6):641-645.

5. Bern C, Montgomery SP, Herwaldt BL, Rassi A Jr, Marin-Neto JA, Dantas RO, Maguire JH, Acquatella H, Morillo C, Kirchhoff LV, et al: Evaluation and treatment of chagas disease in the United States: a systematic review. JAMA 2007, 298(18):2171-2181.

6. Jackson Y, Alirol E, Getaz L, Wolff H, Combescure C, Chappuis F: Tolerance and safety of nifurtimox in patients with chronic chagas disease. Clin Infect Dis 2010, 51(10):e69-e75.

7. Perez-Molina JA, Perez-Ayala A, Moreno S, Fernandez-Gonzalez MC, Zamora J, Lopez-Velez R: Use of benznidazole to treat chronic Chagas' disease: a systematic review with a meta-analysis. J Antimicrob Chemother 2009, 64(6):1139-1147.

8. World Health Organization: Control of Chagas disease. In World Health Organ Tech Rep Ser, vol. 905, 2002/07/03. Brasilia (Brazil): WHO; 2002. i-vi, 1-109, back cover.

9. Viotti R, Vigliano C, Alvarez MG, Lococo B, Petti M, Bertocchi G, Armenti A, De Rissio AM, Cooley G, Tarleton R, et al: Impact of aetiological treatment on conventional and multiplex serology in chronic Chagas disease. PLoS Negl Trop Dis 2011, 5(9):e1314.

10. Rassi A Jr, Rassi A, Marin-Neto JA: Chagas disease. Lancet 2010, 375(9723): 1388-1402.

11. Chappuis F, Mauris A, Holst M, Albajar-Vinas P, Jannin J, Luquetti AO, Jackson $Y$ : Validation of a rapid immunochromatographic assay for diagnosis of Trypanosoma cruzi infection among Latin-American Migrants in Geneva, Switzerland. J Clin Microbiol 2010, 48(8):2948-2952. 
12. Otani MM, Vinelli E, Kirchhoff LV, del Pozo A, Sands A, Vercauteren G, Sabino EC: WHO comparative evaluation of serologic assays for Chagas disease. Transfusion 2009, 49(6):1076-1082.

13. Schijman AG, Bisio M, Orellana L, Sued M, Duffy T, Mejia Jaramillo AM, Cura $C$, Auter F, Veron V, Qvarnstrom Y, et al: International study to evaluate PCR methods for detection of Trypanosoma cruzi DNA in blood samples from Chagas disease patients. PLoS Negl Trop Dis 2011, 5(1):e931.

14. Jackson Y, Getaz L, Wolff H, Holst M, Mauris A, Tardin A, Sztajzel J, Besse V, Loutan L, Gaspoz JM, et al: Prevalence, clinical staging and risk for blood-borne transmission of Chagas disease among Latin American migrants in Geneva, Switzerland. PLoS Negl Trop Dis 2010, 4(2):e592.

15. Berrizbietia M, Ndao M, Gottschalk M, Ache A, Vasquez F, Lacouture S, Medina M, Ward BJ: Development and comparison of enzyme immunoassays for diagnosis of Chagas' disease using fixed forms of Trypanosoma cruzi (Epimastigotes, Amastigotes, and Trypomastigotes) and assessment of antigen stability for the three assays. J Clin Microbiol 2004, 42(4):1766-1769.

16. Ndao M, Kelly N, Normandin D, Maclean JD, Whiteman A, Kokoskin E, Arevalo I, Ward BJ: Trypanosoma cruzi infection of squirrel monkeys: comparison of blood smear examination, commercial enzyme-linked immunosorbent assay, and polymerase chain reaction analysis as screening tests for evaluation of monkey-related injuries. Comp Med 2000, 50(6):658-665.

17. Fabbro DL, Streiger ML, Arias ED, Bizai ML, del Barco M, Amicone NA: Trypanocide treatment among adults with chronic Chagas disease living in Santa Fe city (Argentina), over a mean follow-up of 21 years: parasitological, serological and clinical evolution. Rev Soc Bras Med Trop 2007, 40(1):1-10.

18. Coura JR, de Abreu LL, Willcox HP, Petana W: Comparative controlled study on the use of benznidazole, nifurtimox and placebo, in the chronic form of Chagas' disease, in a field area with interrupted transmission. I. Preliminary evaluation. Rev Soc Bras Med Trop 1997, 30(2):139-144.

19. Cancado JR: Long term evaluation of etiological treatment of chagas disease with benznidazole. Rev Inst Med Trop Sao Paulo 2002, 44(1):29-37.

20. Viotti R, Vigliano C, Armenti H, Segura E: Treatment of chronic Chagas' disease with benznidazole: clinical and serologic evolution of patients with long-term follow-up. Am Heart J 1994, 127(1):151-162.

21. Viotti R, Vigliano C, Lococo B, Bertocchi G, Petti M, Alvarez MG, Postan M, Armenti A: Long-term cardiac outcomes of treating chronic Chagas disease with benznidazole versus no treatment: a nonrandomized trial. Ann Intern Med 2006, 144(10):724-734

22. Marin-Neto JA, Rassi A Jr, Avezum A Jr, Mattos AC, Rassi A, Morillo CA Sosa-Estani S, Yusuf S: The BENEFIT trial: testing the hypothesis that trypanocidal therapy is beneficial for patients with chronic Chagas heart disease. Mem Inst Oswaldo Cruz 2009, 104(Suppl 1):319-324.

23. Brasil PE, De Castro L, Hasslocher-Moreno AM, Sangenis LH, Braga JU: ELISA versus PCR for diagnosis of chronic Chagas disease: systematic review and meta-analysis. BMC Infect Dis 2010, 10:337.

24. Duffy T, Bisio M, Altcheh J, Burgos JM, Diez M, Levin MJ, Favaloro RR, Freilij $\mathrm{H}$, Schijman AG: Accurate real-time PCR strategy for monitoring bloodstream parasitic loads in chagas disease patients. PLoS Negl Trop Dis 2009, 3(4):e419.

25. Schmunis GA, Yadon ZE: Chagas disease: a Latin American health problem becoming a world health problem. Acta Trop 2009, 115(1-2):14-21.

26. Jackson Y, Castillo S, Hammond P, Besson M, Brawand-Bron A, Urzola D, Gaspoz JM, Chappuis F: Metabolic, mental health, behavioural and socioeconomic characteristics of migrants with Chagas disease in a non-endemic country. Trop Med Int Health 2012, 17(5):595-603.

27. Ndao M, Spithill TW, Caffrey R, Li H, Podust VN, Perichon R, Santamaria C, Ache A, Duncan M, Powell MR, et al: Identification of novel diagnostic serum biomarkers for Chagas' disease in asymptomatic subjects by mass spectrometric profiling. J Clin Microbiol 2010, 48(4):1139-1149.

28. Laucella SA, Mazliah DP, Bertocchi G, Alvarez MG, Cooley G, Viotti R, Albareda MC, Lococo B, Postan M, Armenti A, et al: Changes in Trypanosoma cruzispecific immune responses after treatment: surrogate markers of treatment efficacy. Clin Infect Dis 2009, 49(11):1675-1684.

29. Vitelli-Avelar DM, Sathler-Avelar R, Wendling AP, Rocha RD, Teixeira-Carvalho A, Martins NE, Dias JC, Rassi A, Luquetti AO, Eloi-Santos SM, et al: Nonconventional flow cytometry approaches to detect anti-Trypanosoma cruzi immunoglobulin G in the clinical laboratory. $J$ Immunol Methods 2007, 318(1-2):102-112.

30. Fernández-Villegas A, Pinazo MJ, Marañón C, Thomas MC, Posada E, Carrilero B, Segovia M, Gascon J, López MC: Short-term follow-up of chagasic patients after benznidazole treatment using multiple serological markers. BMC Infect Dis 2011, 11:206.

doi:10.1186/1471-2334-13-85

Cite this article as: Jackson et al:: Serological and parasitological response in chronic Chagas patients 3 years after nifurtimox treatment. BMC Infectious Diseases 2013 13:85.

\section{Submit your next manuscript to BioMed Central and take full advantage of:}

- Convenient online submission

- Thorough peer review

- No space constraints or color figure charges

- Immediate publication on acceptance

- Inclusion in PubMed, CAS, Scopus and Google Scholar

- Research which is freely available for redistribution

Submit your manuscript at www.biomedcentral.com/submit
C Biomed Central 